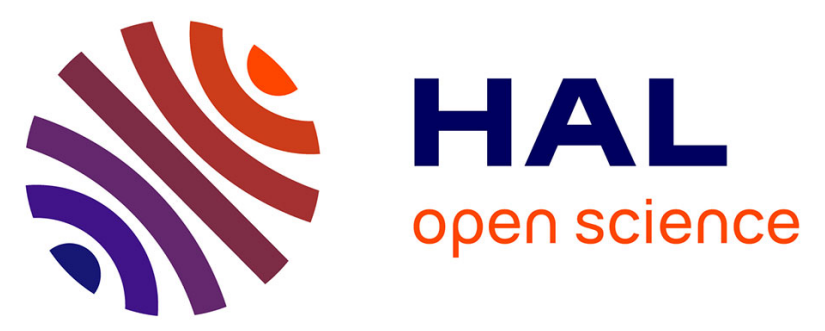

\title{
Influence of radiation heat transfer on parallel hot-wire thermal conductivity measurements of semi-transparent materials at high temperature
}

Léa Penazzi, Yves Jannot, Johann Meulemans, Olivier Farges, Vincent Schick

\section{- To cite this version:}

Léa Penazzi, Yves Jannot, Johann Meulemans, Olivier Farges, Vincent Schick. Influence of radiation heat transfer on parallel hot-wire thermal conductivity measurements of semi-transparent materials at high temperature. International Journal of Thermal Sciences, 2022, 179, pp.107690. 10.1016/j.ijthermalsci.2022.107690 . hal-03517148v2

\section{HAL Id: hal-03517148 \\ https://hal.science/hal-03517148v2}

Submitted on 29 May 2022

HAL is a multi-disciplinary open access archive for the deposit and dissemination of scientific research documents, whether they are published or not. The documents may come from teaching and research institutions in France or abroad, or from public or private research centers.
L'archive ouverte pluridisciplinaire HAL, est destinée au dépôt et à la diffusion de documents scientifiques de niveau recherche, publiés ou non, émanant des établissements d'enseignement et de recherche français ou étrangers, des laboratoires publics ou privés.

\section{(ㅇ)(1) $\$$}

Distributed under a Creative Commons Attribution - NonCommercial - NoDerivatives| 4.0 


\title{
Influence of radiation heat transfer on parallel hot-wire thermal conductivity measurements of semi-transparent materials at high temperature
}

\author{
Léa Penazzi ${ }^{\mathrm{a}, \mathrm{b}, *}$, Yves Jannot ${ }^{\mathrm{a}, \mathrm{b}}$, Johann Meulemans ${ }^{\mathrm{b}, \mathrm{c}}$, Olivier Farges ${ }^{\mathrm{a}, \mathrm{b}}$, Vincent Schick ${ }^{\mathrm{a}, \mathrm{b}}$ \\ ${ }^{a}$ Université de Lorraine, CNRS, LEMTA, F-54500 Vandœuvre-lès-Nancy, France \\ ${ }^{\mathrm{b}}$ Laboratoire Commun Canopée, CNRS, Université de Lorraine, Saint-Gobain, France \\ c Saint-Gobain Research Paris, 39 quai Lucien Lefranc, F-93303 Aubervilliers, France
}

\section{A R T I C L E I N F O}

\section{Keywords:}

Conduction

Radiation

Thermal conductivity

Estimation method

Parallel hot-wire

\begin{abstract}
A B S T R A C T
This paper presents a study of the influence of the radiation transfer on the thermal conductivity measurement in a parallel hot-wire method at high temperature. Simulations of the temperature evolution are first carried out with COMSOL Multiphysics ${ }^{\circledR}$ using the coupled conduction-radiation module based on the P1 approximation for the radiation calculation. A wide range of conditions were investigated: a low and a high density insulator were considered with various radiation characteristics (purely scattering, absorbing and scattering, purely absorbing) and with temperature varying up to $1500^{\circ} \mathrm{C}$. This study enables the determination of the validity limit of the modeling of the temperature by a purely conductive model with an equivalent conductivity based on the Rosseland approximation. When this assumption is valid, a new estimation process was proposed to improve the estimation accuracy of the thermal properties. A calibration process of the distance between the hot-wire and the thermocouple has also been proposed and validated, enabling a more accurate estimation of the volume heat capacity.

An experimental study carried out on three insulating materials with densities ranging from $581 \mathrm{~kg} \mathrm{~m}^{-3}$ to $910 \mathrm{~kg} \mathrm{~m}^{-3}$ and at temperatures ranging from $20^{\circ} \mathrm{C}$ to $1200{ }^{\circ} \mathrm{C}$ confirms the results of the theoretical study. Finally, a method enabling the estimation of the extinction coefficient from thermal conductivity measurements at various temperatures is presented and successfully applied to the three tested materials.
\end{abstract}

\section{Introduction}

As previously pointed out in [1], knowledge of the thermal conductivity of high temperature insulating materials is of great importance for the control of industrial processes. It has also been stated that the parallel hot-wire method is one of the most suitable method to measure the thermal conductivity at high temperature [1,2], moreover it is a standardized method [3]. Some recent developments in the modeling of this method have led to a better accuracy of the measurement $[1,3]$. Nevertheless, the estimation process is more often based on a purely conductive model, considering an equivalent thermal conductivity that is the sum of the thermal conductivity due to conduction and of the radiative thermal conductivity [1-6]. This approximation holds true for optically thick media.

Some authors investigated the effect of radiation heat transfer without making this assumption. Ebert and Fricke [7] simulated the heat transfer in a hot-wire measurement with a one dimensional coupled conduction-radiation model valid only for optically thick media. The radiation emitted by the heating wire was also neglected. Their theoretical study showed that the hot-wire method is applicable only if the extinction coefficient exceeds a critical value. Gross and Tran [8] simulated the heat transfer in a hot-wire measurement with a one dimensional coupled conduction-radiation model solved by the discrete ordinates method. They showed that this critical value increases with the temperature. The influence of the proportion of diffusion/absorption in the extinction coefficient was not addressed in their theoretical study. Coquard et al. [9] developed a two dimensional coupled conduction-radiation model solved by the discrete ordinates method. They used it to study the accuracy of the estimation of the thermal conductivity by the hot-wire method, they highlighted the estimation bias for materials of low density or low optical thickness. Nevertheless, the study was applied to only one material (an EPS foam) and at ambient temperature. Daouas et al. [10] simulated the heat transfer in a parallel hot wire measurement with a one dimensional coupled conduction-radiation model solved by the discrete ordinates method. A sensitivity study showed the possibility of estimating the absorption coefficient in addition to the thermal parameters. However, the study was performed at room temperature on a very weakly

\footnotetext{
* Corresponding author at: Université de Lorraine, CNRS, LEMTA, F-54500 Vandœuvre-lès-Nancy, France.

E-mail address: lea.penazzi@univ-lorraine.fr (L. Penazzi).
} 


\begin{tabular}{|c|c|}
\hline \multicolumn{2}{|c|}{ Nomenclature } \\
\hline \multicolumn{2}{|c|}{ Greek Symbols } \\
\hline$\beta$ & Extinction coefficient $\left(\mathrm{m}^{-1}\right)$ \\
\hline$\lambda$ & $\begin{array}{l}\text { Thermal conductivity of the considered } \\
\text { material }\left(\mathrm{W} \mathrm{m}^{-1} \mathrm{~K}^{-1}\right)\end{array}$ \\
\hline$\Omega$ & Solid angle (sr) \\
\hline$\phi$ & Heat flux density $\left(\mathrm{W} \mathrm{m}^{-2}\right)$ \\
\hline$\rho$ & Density of the material $\left(\mathrm{kg} \mathrm{m}^{-3}\right)$ \\
\hline$\sigma$ & Stefan-Boltzmann constant $\left(\mathrm{W} \mathrm{m}^{-2} \mathrm{~K}^{-4}\right)$ \\
\hline$\tau$ & Optical thickness (-) \\
\hline$\theta$ & Laplace transform of $T(d, t)$ \\
\hline$\varepsilon$ & Emissivity (-) \\
\hline$\varphi_{\mathrm{w}}$ & $\begin{array}{l}\text { Linear heat flux produced by the heating } \\
\text { wire }\left(\mathrm{W} \mathrm{m}^{-1}\right)\end{array}$ \\
\hline$\omega$ & Direction $(-)$ \\
\hline \multicolumn{2}{|c|}{ Latin Symbols } \\
\hline $\mathcal{I}$ & Radiant intensity $\left(\mathrm{W} \mathrm{m}{ }^{-2} \mathrm{sr}^{-1}\right.$ ) \\
\hline$n$ & Outward unit direction on the surface $(-)$ \\
\hline$a$ & $\begin{array}{l}\text { Thermal diffusivity of the isotropic mate- } \\
\text { rial }\left(\mathrm{m}^{2} \mathrm{~s}^{-1}\right)\end{array}$ \\
\hline$c$ & Heat capacity of the material $\left(\mathrm{J} \mathrm{kg}^{-1} \mathrm{~K}^{-1}\right)$ \\
\hline$d$ & $\begin{array}{l}\text { Distance between the heating wire and the } \\
\text { thermocouple }(\mathrm{m})\end{array}$ \\
\hline$G$ & Incident radiation $\left(\mathrm{W} \mathrm{m}^{-2}\right)$ \\
\hline$I$ & $\begin{array}{l}\text { Electrical intensity passing through the } \\
\text { heating wire (A) }\end{array}$ \\
\hline$k_{\mathrm{a}}$ & Absorption coefficient $\left(\mathrm{m}^{-1}\right)$ \\
\hline$k_{\mathrm{s}}$ & Scattering coefficient $\left(\mathrm{m}^{-1}\right)$ \\
\hline$K_{i}$ & $\begin{array}{l}\text { Modified Bessel functions of the second } \\
\text { kind } i \in\{0 ; 1\}\end{array}$ \\
\hline$l_{\mathrm{w}}$ & Length of the heating wire $(\mathrm{m})$ \\
\hline$N$ & Stark number $(-)$ \\
\hline$n$ & Refractive index $(-)$ \\
\hline$P$ & Phase function $(-)$ \\
\hline$p$ & Laplace parameter $\left(\mathrm{s}^{-1}\right)$ \\
\hline$Q_{\mathrm{r}}$ & Radiative heat source $\left(\mathrm{W} \mathrm{m}^{-3}\right)$ \\
\hline$R$ & $\begin{array}{l}\text { Thermal contact resistance between the } \\
\text { heating wire and the sample }\left(\mathrm{K} \mathrm{W}^{-1}\right)\end{array}$ \\
\hline$r_{\mathrm{w}}$ & Radius of the heating wire $(\mathrm{m})$ \\
\hline$R_{\mathrm{el}}$ & Electrical resistance of the heating wire $(\Omega)$ \\
\hline$T$ & Temperature (K) \\
\hline$U$ & Voltage at the heating wire terminals $(\mathrm{V})$ \\
\hline \multicolumn{2}{|c|}{ Subscripts } \\
\hline c & Relating to the conductive transfer (-) \\
\hline $\mathrm{r}$ & Relating to the radiative transfer $(-)$ \\
\hline $\mathrm{w}$ & Relating to the hot wire $(-)$ \\
\hline $\exp$ & Relating to the experimentation (-) \\
\hline $\bmod$ & Relating to the modeling $(-)$ \\
\hline
\end{tabular}

absorbing and non-scattering material. Zhang et al. [11] performed a numerical study simulating the coupled conduction-radiation heat transfer in a purely absorbing medium in both cases of hot-wire and parallel hot-wire measurements with discrete ordinates method. The numerical analysis shows that the thermal conductivity of insulated material with low extinction coefficient measured by hot-wire device is overestimated at high temperature. However, the study was conducted on only one material with unique density and thermal conductivity. The chosen thermal conductivity value was very low $\left(\lambda=0.02 \mathrm{~W} \mathrm{~m}^{-1} \mathrm{~K}^{-1}\right)$ and the maximum temperature does not exceed $1000{ }^{\circ} \mathrm{Celsius}$.

The purpose of this study is to establish the conditions under which the temperature evolution in a parallel hot-wire measurement can be correctly represented by a pure conductive model using an effective thermal conductivity. A theoretical study using the P1 approximation method implemented in COMSOL Multiphysics ${ }^{\circledR}$ will be performed for temperatures ranging from ambient to $1500^{\circ} \mathrm{C}$, for both absorbing and scattering media and for several densities, extending the range of materials and temperatures of the previous studies. When the purely conductive model applies, a new estimation method is proposed that improves both thermal conductivity and thermal capacity estimations. A calibration process of the distance between the hot wire and the thermocouple will also be proposed, enabling a more accurate estimation of the volumetric heat capacity. The results will be supported by an experimental study carried out on three insulating materials with densities ranging from $581 \mathrm{~kg} \mathrm{~m}^{-3}$ to $910 \mathrm{~kg} \mathrm{~m}^{-3}$ and at temperatures ranging from $20^{\circ} \mathrm{C}$ to $1200^{\circ} \mathrm{C}$. Finally, a method enabling the estimation of the extinction coefficient from thermal conductivity measurements at various temperatures is presented and successfully applied to the three tested materials.

\section{Principle and models}

\subsection{Principle}

The schematic diagram of the classical parallel hot-wire set-up is presented in Fig. 1. A resistive heating wire with a radius $r_{\mathrm{w}}$ is inserted in a groove placed on the surface of the bottom sample. A thermocouple is inserted in another groove placed at the surface of the same sample at distance $d$ of the heating wire. A second sample with the same dimensions as the bottom sample is then placed on top of it. A constant heat flow rate is then imposed in the heating wire by passing an electrical current with a constant intensity $I$. The equations of the system are:

$\begin{cases}\rho c \frac{\partial T}{\partial r t}=\nabla \cdot\left(\lambda_{\mathrm{c}} \nabla T\right) & \text { (a) } \\ T(r, 0)=0 & \text { (b) } \\ \lim _{r \rightarrow \infty} T(r, 0)=0 & \text { (c) } \\ \varphi_{w}=\rho_{\mathrm{w}} c_{\mathrm{w}} \pi r_{\mathrm{w}}^{2} \frac{\partial T_{\mathrm{w}}}{\partial t}+\frac{T_{\mathrm{w}}-T\left(r_{\mathrm{w}}\right)}{R l_{\mathrm{w}}} & \text { (d) }\end{cases}$

A complete model of the system can be found in [1], it is based on the following hypotheses:

- The samples are infinite in the $O x$ and $O z$ directions (semi-infinite medium hypothesis).

- There is no temperature gradient along the $O y$ direction ("long wire").

- The heat flow rate $\varphi_{\mathrm{w}}$ produced in the heating wire is constant.

- The mass of the thermocouple is negligible.

- Radiation heat transfer is negligible or may be represented by an effective thermal conductivity.

Jannot and Degiovanni [1] established the following expression for the Laplace transform of the thermocouple temperature:

$$
=\frac{\theta(d, p)}{\rho_{\mathrm{w}} c_{\mathrm{w}} \pi r_{\mathrm{w}}^{2} p K_{0}\left(\sqrt{\frac{p}{a}} r_{\mathrm{w}}\right)+2 \pi \lambda_{\mathrm{c}} \sqrt{\frac{p}{a}} r_{\mathrm{w}}\left[1+\rho_{\mathrm{w}} c_{\mathrm{w}} \pi r_{\mathrm{w}}^{2} p R l_{\mathrm{w}}\right] K_{1}\left(\sqrt{\frac{p}{a}} r_{\mathrm{w}}\right)}
$$

The temperature rise $T(d, t)$ is calculated by applying an inverse Laplace transform to (2) using the De Hoog algorithm [12]. 


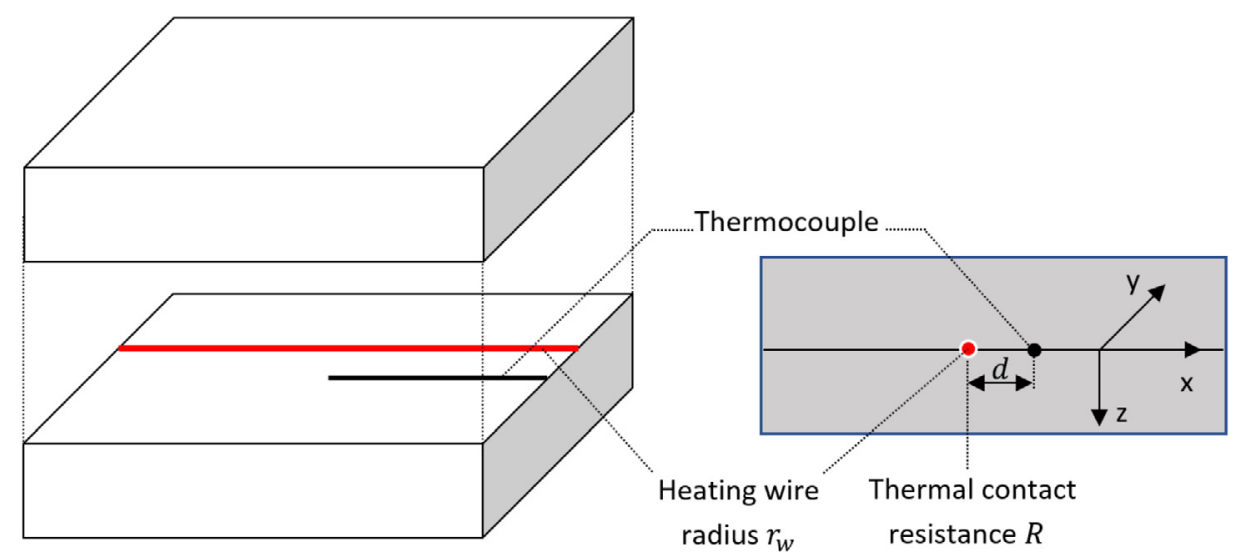

Fig. 1. Schematic view of a parallel hot-wire set-up.

\subsection{General equations}

It is assumed here that the medium is gray. The coupled conduction and radiation heat transfer in transient hot-wire measurements is described by the following energy balance:

$\rho c \frac{\partial T}{\partial t}=-\nabla \cdot\left(\phi_{\mathrm{c}}+\boldsymbol{\phi}_{\mathrm{r}}\right)$

Where $\boldsymbol{\phi}_{\mathrm{c}}$ is the conductive heat flux given by (4) and $\boldsymbol{\phi}_{\mathrm{r}}$ is the radiative flux given by (4).

$$
\left\{\begin{array}{l}
\phi_{\mathrm{c}}=-\lambda_{\mathrm{c}} \frac{\partial T}{\partial \mathrm{r}} \\
\boldsymbol{\nabla} \cdot \boldsymbol{\phi}_{\mathrm{r}}=4 k_{\mathrm{a}} n^{2} \sigma T^{4}-k_{\mathrm{a}} \int_{4 \pi} \mathcal{I}(\omega) \mathrm{d} \Omega
\end{array}\right.
$$

$\mathcal{I}(\omega)$ is the radiant intensity in the $\omega$ direction given by the radiative transfer equation (RTE) (Eq. (5)):

$$
\begin{aligned}
\frac{\partial \mathcal{I}(\omega)}{\partial s}= & -\left(k_{\mathrm{a}}+k_{\mathrm{s}}\right) \mathcal{I}(\boldsymbol{\omega})+k_{\mathrm{a}} n^{2} \sigma T^{4} \\
& +\frac{k_{\mathrm{s}}}{4 \pi} \int_{4 \pi} \mathcal{I}\left(\boldsymbol{\omega}^{\prime}\right) P\left(\boldsymbol{\omega}^{\prime} \rightarrow \boldsymbol{\omega}\right) \mathrm{d} \Omega^{\prime}
\end{aligned}
$$

Where $k_{\mathrm{a}}$ is the absorption coefficient, $k_{\mathrm{s}}$, the scattering coefficient, $n$, the refractive index and $\sigma$ is the Stefan-Boltzmann constant.

\subsection{Model M1: P1 approximation}

The P1 approximation, corresponding to a spherical harmonics method (PN) truncated at the first order, enables solving the RTE presented in (5). The P1 approximation relies on the assumption that the scattering is linear isotropic [13]. This assumption is justified in this present configuration as the medium is optically thick, $\tau \gg 1$, where $\tau$ is the optical thickness defined by the integral of the extinction coefficient $\beta$ along a typical optical path $\tau=\int_{0}^{s} \beta \mathrm{d} s$.

From a computational point of view this approximation has a limited impact because it introduces only one additional degree of freedom for the incident radiation $G\left(\mathrm{~W} \mathrm{~m}{ }^{-2}\right)$, which is a scalar quantity and adds a heat source or sink to the temperature equation to account for radiative heat transfer contributions. This method, however, fails to accurately represent cases where the radiative intensity propagation dominates over its diffusivity or where the scattering effects cannot be described by a linear isotropic phase function. The P1 approximation is a simplified approach, which is expected to be fairly good for absorbing and highly scattering media at large optical distances from boundaries or interfaces that have a strong variation of temperature and radiative characteristics of the medium [14]. The P1 approximation solves the RTE equation, expressed in (5), by solving the following equation:

$\nabla \cdot\left(-D_{P 1} \nabla G\right)=-Q_{\mathrm{r}}$

where $G$ is the incident radiation (Eq. (7)), $D_{P 1}$ is the P1 diffusion coefficient in the case of an isotropic diffusion (Eq. (7)) and $Q_{\mathrm{r}}$ is the radiative volumetric heat source (Eq. (7)).

$\begin{cases}G=\int_{0}^{4 \pi} \mathcal{I}(\boldsymbol{\omega}) \mathrm{d} \Omega & \text { (a) } \\ D_{P 1}=\frac{1}{3 k_{\mathrm{a}}+3 k_{\mathrm{s}}} & \text { (b) } \\ Q_{\mathrm{r}}=\boldsymbol{\nabla} \cdot \boldsymbol{\phi}_{\mathrm{r}}=k_{\mathrm{a}}\left(G-4 k_{\mathrm{a}} n^{2} \sigma T^{4}\right) & \text { (c) }\end{cases}$

Regarding the boundary conditions, at the hot wire surface, thermocouples surfaces and sample external surfaces are considered as opaque surfaces. The following equation accounts for the radiation emitted/absorbed by the boundaries and is known as the Marshak's boundary condition [15]:

$\boldsymbol{n} \cdot\left(-D_{P 1} \nabla G\right)=\phi_{\mathrm{r}, \text { net }}$

Where $\phi_{\mathrm{r}, \text { net }}$ is the net radiative heat flux at the boundary and $n$ the outward unit direction on the surface. In the following simulations, surfaces of the hot wire, thermocouples and the external surface of the sample were considered gray with an approximate common emissivity value of $\varepsilon=0.8$. The net radiative heat flux is then expressed as follows:

$\phi_{\mathrm{r}, \text { net }}=\frac{\varepsilon}{2(2-\varepsilon)}\left(4 n^{2} \sigma T^{4}-G\right)$

\subsection{Model M2: Rosseland approximation}

If the medium is gray and optically thick, the radiative flux divergence is expressed as follows:

$\phi_{\mathrm{r}}=-\lambda_{\mathrm{r}} \frac{\partial T}{\partial r}$

With the expression of the radiative thermal conductivity [16]:

$\lambda_{\mathrm{r}}(T)=\frac{16}{3} \frac{n^{2} \sigma T^{3}}{\beta}$

where $\beta$ is the extinction coefficient of the medium given by $\beta=$ $k_{\mathrm{a}}+k_{\mathrm{s}}$. We are thus brought back to a pure conduction problem with an effective conductivity $\lambda=\lambda_{\mathrm{c}}+\lambda_{\mathrm{r}}$.

\section{Materials and experimental device}

An experimental study was carried out to confirm the theoretical results. Measurements were undertaken on an isotropic material: SilPower ${ }^{\circledR}$ (Saint-Gobain Quartz) with three different densities: 581, 741 and $910 \mathrm{~kg} \mathrm{~m}^{-3}$ referenced respectively as $S G, M G$ and $H G$. SilPower ${ }^{\circledR}$ Rigid Silica is a high performance infra-red reflector obtained from Quartzel ${ }^{\circledR}$ fibers having an $\mathrm{SiO}_{2}$ content $\geq 99.95 \%$ (Saint-Gobain, 2021).

The dimensions of the samples are $230 \times 120 \times 60 \mathrm{~mm}^{3}$ as recommended by the standard ISO 8894-2:2007. The parallel hot-wire (PHW) measurements were conducted at 20, 200, 400, 600, 800, 1000 and 


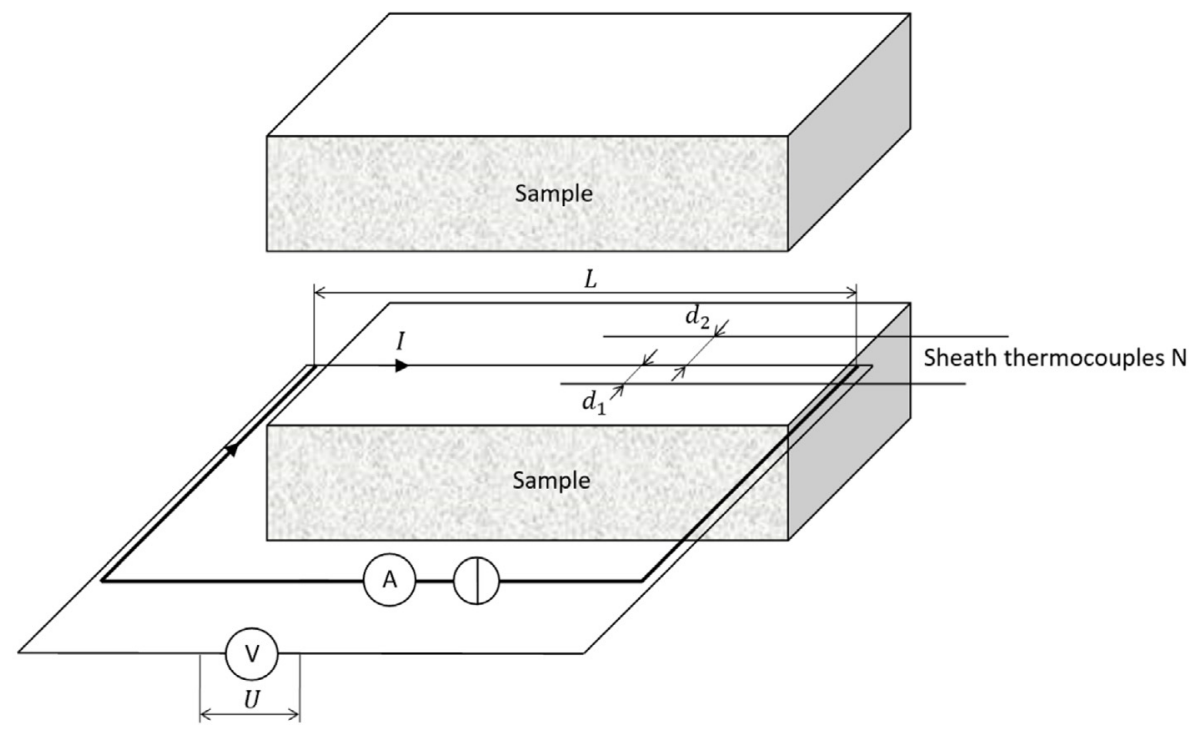

Fig. 2. Schematic view of the measurement set-up.

$1200^{\circ} \mathrm{C}$. Three experiments were carried out for each temperature and for each density. Fig. 2 presents a schematic view of the measurement set-up. The heating wire made of Nickel-Chrome $80 / 20$ has a diameter of $0.5 \mathrm{~mm}$ and it is inserted in a groove with a $0.5 \times 0.5 \mathrm{~mm}^{2}$ section. The groove with the same length as the sample was machined in the middle of one face of the sample.

To study the influence of the distance between the heating wire and the thermocouple, the temperature rise was measured at two locations, respectively at $d_{1} \approx 10 \mathrm{~mm}$ and $d_{2} \approx 15 \mathrm{~mm}$ from the heating wire. The temperature measurements were carried out with two type $N$ sheath thermocouples with an outer diameter of $0.5 \mathrm{~mm}$, inserted in grooves with a $0.5 \times 0.5 \mathrm{~mm}^{2}$ section machined on the half-length of the sample in order to measure the temperature at the center. The temperature is recorded with $0.1 \mathrm{~s}$ time step by a data logger Picolog TC08. The standard deviation of the temperature measurement is $0.01{ }^{\circ} \mathrm{C}$ before heating.

The device was placed in an electric fusing kiln (Vecstar furnace) equipped with a temperature controller (Watlow EZ-Zone) ensuring a very good temperature stability inside the kiln $\left( \pm 1^{\circ} \mathrm{C}\right)$.

Two wires were point-welded on the heating wire with a distance $l_{\mathrm{w}}=235 \mathrm{~mm}$ between the two weldings (see Fig. 2). These wires were connected to a voltmeter (Almemo 2890-9) to measure the electrical voltage $U$ between the two points. A stabilized power supply (Tektronix PWS2185) produces an electrical current with a constant intensity $I$ passing through the wire. These measurements allow the calculation of the electrical resistance per meter of the heating wire with the following formula:

$$
\frac{R_{\mathrm{el}}}{l_{\mathrm{w}}}=\frac{U}{I l_{\mathrm{w}}}
$$

\section{Estimation method}

In previous works [1,3], the Levenberg-Marquardt algorithm [17] was used to find the values of the unknown parameters $\lambda, \rho c$ and $R$ that minimize the sum of the quadratic errors:

$S=\sum_{n=1}^{N}\left[T_{\exp }(d, t)-T_{\bmod }(d, t)\right]^{2}$

Where $T_{\text {mod }}$ is calculated by inverse Laplace transform of (2). Using this estimation method, it was found that the estimated values of the thermal contact resistance $R$ become negative when the temperature increases which does not make any physical sense. As an example, Fig. 3(a) represents: the experimental curve and the model curve plotted with the triplet $(\lambda, \rho c, R)$ of the estimated values and the residuals (multiplied by 10 for a better reading) for an experiment on the SilPower ${ }^{\circledR} \mathrm{SG}$ at $600^{\circ} \mathrm{C}$ for which the estimation over the interval $[100 \mathrm{~s}, 900 \mathrm{~s}]$ led to the value $R=-5.8 \mathrm{~K} \cdot \mathrm{W}^{-1}$. It can be seen that the estimation residuals are perfectly flat over the estimation interval which means that from $100 \mathrm{~s}$ onwards, the Rosseland assumption is verified, hence, in this case, the use of the model is validated. Between $0 \mathrm{~s}$ and $100 \mathrm{~s}$ (see Fig. 3(b)), the experimental temperature is higher than the model temperature which means that the heat flux transmitted by radiation is higher than that predicted by the model. The negative value of the contact resistance compensates for this model bias and thus has the effect of shifting the temperature so that is obtained $T_{\exp }(100 \mathrm{~s})=T_{\text {mod }}(100 \mathrm{~s})$. However, it has another undesirable effect illustrated in Fig. 4, which depicts the reduced temperature sensitivity to the contact resistance for different values of the contact resistance. The value $R=2 \mathrm{~K} \cdot \mathrm{W}^{-1}$ is an average value consistent with those obtained from COMSOL Multiphysics ${ }^{\circledR}$ simulations of our device. We can see that the sensitivity to $R$ which varies little with the value $R=2 \mathrm{~K} \cdot \mathrm{W}^{-1}$ varies significantly for strongly negative values of $R$. We will show that this can create an estimation bias on the values of $\lambda$ and $\rho c$.

To do so, we performed a simulation based on Eq. (2) with the estimated values $\lambda=0.22 \mathrm{~W} \mathrm{~m}^{-1} \mathrm{~K}^{-1}$ and $\rho c=6.11 \times 10^{5} \mathrm{~J} \mathrm{~m}^{-3} \mathrm{~K}^{-1}$ and considering $R=2 \mathrm{~K} \mathrm{~W}^{-1}$, and then we added to the result a constant value $T_{0}$. This $T_{0}$ value thus represents the contribution of radiation between 0 and $100 \mathrm{~s}$ not taken into account by the Rosseland model.

Two estimation methods were then applied to this curve over the interval [100s, $800 \mathrm{~s}]$ :

- An estimation E1 of $(\lambda, \rho c, R)$ as described above.

- An estimation E2 of $\left(\lambda, \rho c, T_{1}\right)$ by calculating the temperature from the (2), considering $R=0 \mathrm{~K} \cdot \mathrm{W}^{-1}$ and adding a constant value $T_{1} T_{0}$ that is:

$$
T(d, t)=\mathcal{L}^{-1}\left[\frac{\frac{\varphi_{\mathrm{w}}}{p L} K_{0}\left(\frac{p}{a} d\right)}{\rho_{\mathrm{w}} c_{\mathrm{w}} \pi r_{\mathrm{w}}{ }^{2} p K_{0}\left(\frac{p}{a} r_{\mathrm{w}}\right)+2 \pi \lambda \frac{p}{a} r_{\mathrm{w}} K_{1}\left(r \frac{p}{a}\right)}\right]+T_{1} T_{0}
$$




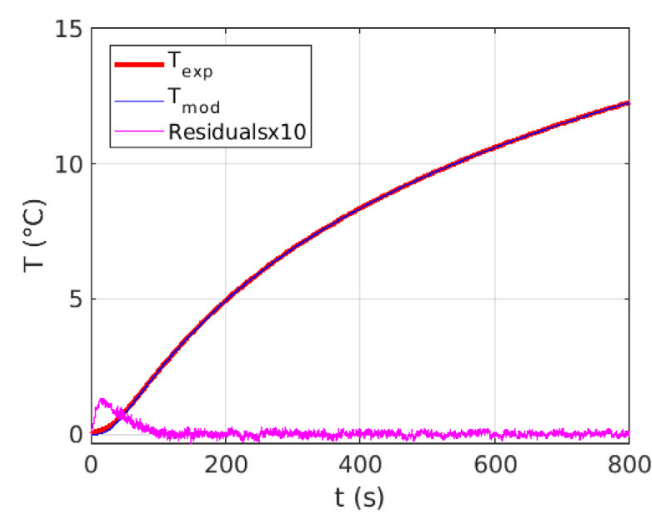

(a) From $0 \mathrm{~s}$ to $800 \mathrm{~s}$

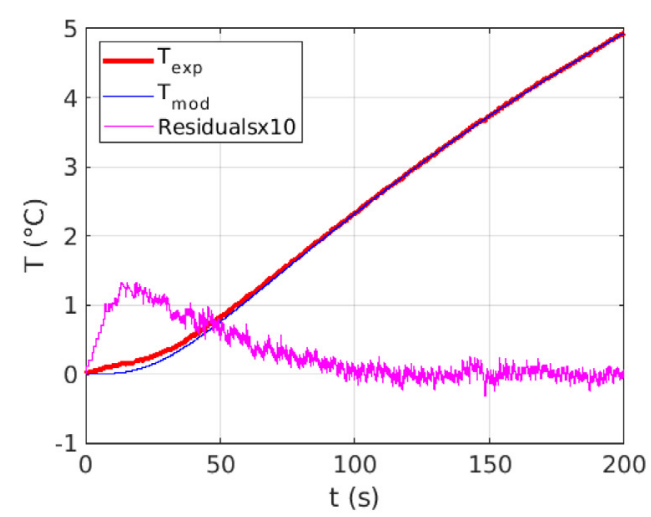

(b) From $0 \mathrm{~s}$ to $200 \mathrm{~s}$

Fig. 3. Experimental and simulated curves with estimation residuals $\times 10$ for an experiment with SilPower ${ }^{\circledR} \mathrm{SG}$ at $600{ }^{\circ} \mathrm{C}$.

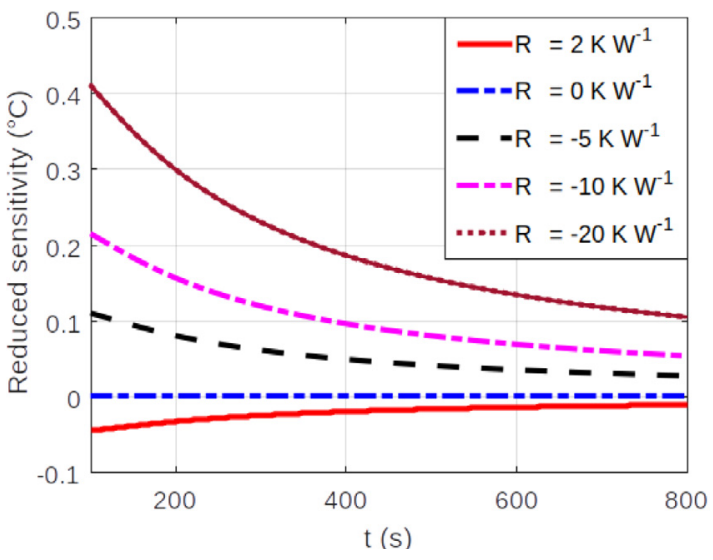

Fig. 4. Reduced sensitivity of the temperature according to thermal contact resistance $R$ values $\left(\lambda=0.22 \mathrm{~W} \mathrm{~m}^{-1} \mathrm{~K}^{-1} ; \rho c=6.11 \times 10^{5} \mathrm{~J} \mathrm{~m}^{-3} \cdot \mathrm{K}^{-1}\right)$.

Where $\mathcal{L}^{-1}$ is the operator of the inverse Laplace transform. $T_{0}$ and $T_{1}$ both stand for the contribution of radiation between 0 and 100s. For the sake of rigor though, these values have been differentiated since $T_{0}$ represents the value added to the preceding equation and $T_{1}$ represents the estimated value of $T_{0}$ with the E2 estimation method. Furthermore, one may state that due to the larger contribution of radiative heat transfer at high temperature, the contact resistance $R$ is greatly reduced to approximately zero.

Table 1 shows the estimation results for different values of $T_{0}$. It can be observed that the E2 estimation method leads to a constant error of $0.7 \%$ on $\lambda$ and a negligible error on $\rho c$. The E1 method leads to a small estimation error on $\lambda$ (less than $1 \%$ if $T_{0}<0.75^{\circ} \mathrm{C}$ ) but a large error on $\rho c$ that increases with $T_{0}$. We therefore treated the experimental curves using the E2 estimation method and choosing an estimation interval such that the residuals are flat and centered on zero over this interval.

\section{Validity of the rosseland approximation}

To determine the validity limits of Rosseland approximation in the case of the parallel hot-wire method, we performed simulations using the COMSOL Multiphysics ${ }^{\circledR}$ coupled conduction-radiation transfer solver based on the approximate model P1 [18].

Conduction is modeled with the energy balance equation with a radiative source term (Eqs. (6) to (8)). The radiative source term is modeled with the P1 approximation (Eqs. (11) to (15)). The solution of the transient coupled conduction and radiation equations is solved iteratively by the Finite Element method (FEM). Solid domains formed by the hot wire, the two thermocouples and the sample are meshed
Table 1

Estimated values of the parameters by the two methods E1 and E2 for different values of the temperature step due to radiation between $0 \mathrm{~s}$ to $100 \mathrm{~s}$ with $\lambda=0.22 \mathrm{~W} \mathrm{~m}^{-1} \mathrm{~K}^{-1}$, $\rho c=6.11 \times 10^{5} \mathrm{~J} \mathrm{~m}^{-3} \mathrm{~K}^{-1}$

\begin{tabular}{|c|c|c|c|c|c|c|c|c|c|}
\hline & $\mathrm{T}_{0}$ & ${ }^{\circ} \mathrm{C}$ & 0 & 0.25 & 0.5 & 0.75 & 1.0 & 1.25 & 1.5 \\
\hline \multirow{3}{*}{ E1 } & $\lambda$ & $\mathrm{W} \mathrm{m^{-1 }} \mathrm{K}^{-1}$ & 0.220 & 0.219 & 0.219 & 0.218 & 0.217 & 0.217 & 0.216 \\
\hline & $\rho c\left(\times 10^{5}\right)$ & $\mathrm{J} \mathrm{m}^{-3} \mathrm{~K}^{-1}$ & 6.11 & 5.91 & 5.72 & 5.55 & 5.36 & 5.18 & 5.01 \\
\hline & $R$ & $\mathrm{~K} \mathrm{~W}^{-1}$ & 2.0 & -4.0 & -9.8 & -15.4 & -20.8 & -26.0 & -31.3 \\
\hline \multirow{3}{*}{ E2 } & $\lambda$ & $\mathrm{W} \mathrm{m}^{-1} \mathrm{~K}^{-1}$ & & & & 0.219 & & & \\
\hline & $\rho c\left(\times 10^{5}\right)$ & $\mathrm{J} \mathrm{m}^{-3} \mathrm{~K}^{-1}$ & & & & 6.11 & & & \\
\hline & $T_{1}$ & ${ }^{\circ} \mathrm{C}$ & -0.02 & 0.23 & 0.48 & 0.73 & 0.98 & 1.23 & 1.48 \\
\hline
\end{tabular}

with 4974 unstructured tetrahedrals. The maximum size of an element size is $6.36 \times 10^{-3} \mathrm{~m}$ and the minimum size of an element is $3.6 \times 10^{-5} \mathrm{~m}$. The mesh is automatically created and adapted for the model's physics settings. The time dependency in the FEM is solved by an implicit BDF (Backward Differentiation Formula) method. This implicit solver uses an backward differentiation formulas with order of accuracy varying from one (also known as the backward Euler method) to two. The time step used for the simulation is $0.1 \mathrm{~s}$.

We first compared the solutions given by COMSOL Multiphysics ${ }^{\circledR}$ with the exact solution in two asymptotic cases where an analytical solution exists as proposed by Gross and Tran [8]:

(1) The case where the extinction coefficient is very large $\left(k_{\mathrm{a}}=\right.$ $k_{\mathrm{s}}=10^{8} \mathrm{~m}^{-1}$ ), the temperature evolves as in the case of a purely conductive transfer because $\lambda_{\mathrm{r}}=0 \mathrm{~W} \cdot \mathrm{m}^{-1} \cdot \mathrm{K}^{-1}$.

(2) The case where the material is completely transparent $\left(k_{\mathrm{a}}=k_{\mathrm{s}}=\right.$ $0 \mathrm{~m}^{-1}$ ) with perfectly opaque and reflecting surfaces (the heating wire, thermocouple and external border of the sample with $\epsilon=0$ ) ( non-participant medium).

The geometry considered is a cylinder with an external diameter of $50 \mathrm{~mm}$ heated uniformly on its internal diameter of $0.5 \mathrm{~mm}$, both surfaces being opaque and insulated, and the initial uniform temperature being equal to $1000^{\circ} \mathrm{C}$.

As an example, Fig. 5(a) represents the case of the transparent material the curves of the temperature at a distance $d=15 \mathrm{~mm}$ from the heating wire, obtained with COMSOL Multiphysics ${ }^{\circledR}$ on the one hand and with the exact analytical model on the other hand for the following values of the parameters: $\lambda_{\mathrm{c}}=0.5 \mathrm{~W} \cdot \mathrm{m}^{-1} \cdot \mathrm{K}^{-1}, \rho c=$ $10^{6} \mathrm{~J} \cdot \mathrm{m}^{-3} \cdot \mathrm{K}^{-1}$. The curves are in very good agreement and estimates made from the curves simulated with COMSOL Multiphysics ${ }^{\circledR}$ using the ISO 8894-2:2007 method [2] lead to values of $\lambda$ that differ by less than $0.5 \%$ from the nominal value as shown in Fig. 5(b). The ISO 88942:2007 method [2] was detailed in a previous paper [1], it recommends the estimation for values of the ratio $T(2 t) / T(t)$ between 1.5 and 2.4, 


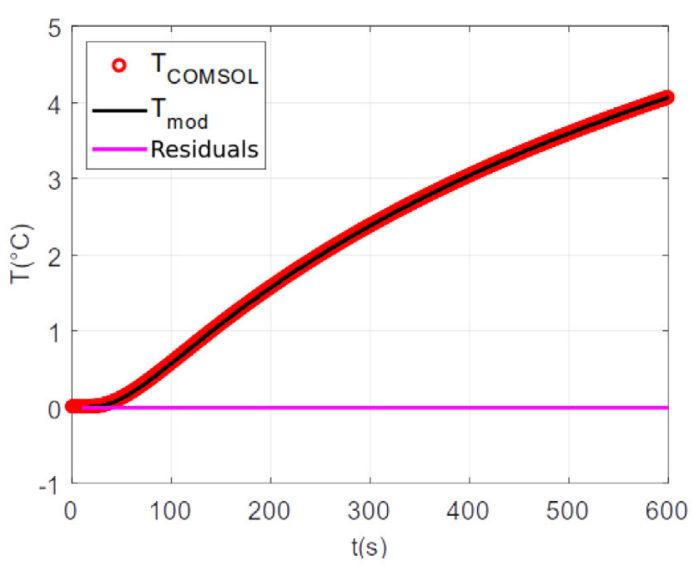

(a)

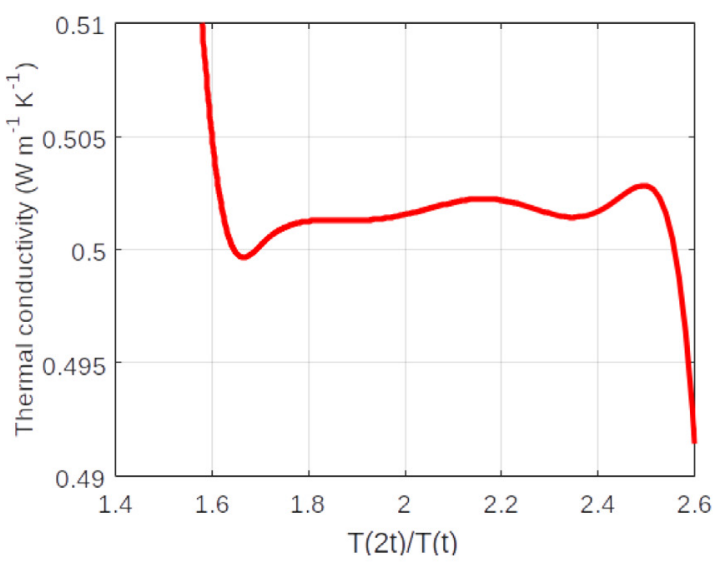

(b)

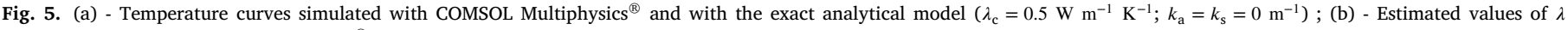
by processing the COMSOL Multiphysics ${ }^{\circledR}$ curve with the standard method ISO 8894-2:2007.

in the presented case the validity limit of the semi-infinite medium assumption reduces this interval to [1.7,2.4]. The results obtained in the case where the extinction coefficient is very large are similar.

After this validation phase, we performed simulations with COMSOL Multiphysics ${ }^{\circledR}$ of the experimental setup shown in Fig. 6. For each simulation, the temperatures of the heating wire and of the two thermocouples placed respectively at $10 \mathrm{~mm}$ and $15 \mathrm{~mm}$ from the heating wire were recorded.

Preliminary tests were carried out in order to ensure the simulations results independence to temporal and spatial discretization. The simulations were performed between $0 \mathrm{~s}$ to $1000 \mathrm{~s}$ :

- For two temperatures: $1000^{\circ} \mathrm{C}$ and $1500^{\circ} \mathrm{C}$.

- For two typical materials: a low density thermal insulating material $\left(\lambda_{\mathrm{c}}=0.1 \mathrm{~W} \cdot \mathrm{m}^{-1} \cdot \mathrm{K}^{-1}\right.$ and $\left.\rho c=2.5 \times 10^{5} \mathrm{~J} \cdot \mathrm{m}^{-3} \cdot \mathrm{K}^{-1}\right)$ and a high density thermal insulating material $\left(\lambda_{\mathrm{c}}=0.5 \mathrm{~W} \cdot \mathrm{m}^{-1} \cdot \mathrm{K}^{-1}\right.$ and $\rho c=10^{6} \mathrm{~J} \cdot \mathrm{m}^{-3} \cdot \mathrm{K}^{-1}$ ).

- For four values of the extinction coefficient $\beta$, ie $500 \mathrm{~m}^{-1}, 1000 \mathrm{~m}^{-1}$, $5000 \mathrm{~m}^{-1}$ and $10000 \mathrm{~m}^{-1}$ and for each value of $\beta$ the following three values of $k_{\mathrm{a}}$ were considered: $k_{\mathrm{a}}=0.0001 \times \beta ; 0.5 \times \beta ; \beta$ (with $k_{\mathrm{s}}=\beta-k_{\mathrm{a}}$ ). The extreme cases correspond respectively to pure scattering and pure absorption.

The absorption coefficient was not chosen equal to zero because it has been shown [19] that in this case the radiative transfer could be represented by a parallel resistance between the two surfaces, which would invalidate the semi-infinite medium hypothesis even at short times. We chose a minimum value equal to $0.0001 \times \beta$.

The refractive index of the medium was chosen equal to unity and the emissivities of the wire and of the thermocouples equal to 0.8 . The thermal properties of the heating wire and thermocouple are those of a stainless steel: $\lambda=11 \mathrm{~W} \cdot \mathrm{m}^{-1} \cdot \mathrm{K}^{-1}$ and $\rho c=3.7 \times 10^{6} \mathrm{~J} \cdot \mathrm{m}^{-3} \cdot \mathrm{K}^{-1}$. The outer surface of the sample was assumed to be opaque, black and insulated.

The temperature curves obtained for the two thermocouples were treated as numerical experiments to estimate the values of the parameters $\lambda_{\text {est }}, \rho c_{\text {est }}$ and $T_{1}$, using the E2 estimation method described previously. The time interval over which the estimation was performed was adjusted for each curve to obtain flat and zero-centered estimation residuals over this interval. The estimated value $\lambda_{\text {est }}$ was then compared to the effective conductivity calculated by:

$\lambda=\lambda_{\mathrm{c}}+\frac{16}{3} \frac{\sigma \bar{T}^{3}}{\beta}$

Where $\lambda_{\mathrm{c}}$ is the phonic conductivity used in the COMSOL Multiphysics ${ }^{\circledR}$ simulation $\beta$ is the extinction coefficient, and $\bar{T}$ is the average temperature of the measurement point as recommended by Gross and Tran [8]. The estimated value $\rho c_{\text {est }}$ was also compared to the nominal value $\rho c$. The analysis of all the results showed that there is no significant difference between the values estimated with $d_{1}=10 \mathrm{~mm}$ and those estimated with $d_{2}=15 \mathrm{~mm}$. Fig. 7 shows the relative deviations between the values of effective conductivity $\lambda_{\text {est }}$ estimated from the temperature at $15 \mathrm{~mm}$ and the nominal values calculated by (15) as a function of the Stark number defined by Viskanta [20] and Cintolesi et al. [21] and presented in (16):

$N=\frac{\beta \lambda_{\mathrm{c}}}{4 \sigma \bar{T}^{3}}$

It is proportional to the ratio of the thermal conductivity $\lambda$ to the radiative conductivity $\lambda_{\mathrm{r}}$.

Fig. 7(a) shows that in the case of the PHW method for the estimation error to be less than $2 \%$ it is necessary that:

- $N>0.1$ in the case of an absorbing and scattering material, see Fig. 7(a).

- $N>2$ in the case of a purely scattering material, this limit value can be lower for materials of low thermal conductivity $(N>0.8$ for $\lambda=0.1 \mathrm{~W} \cdot \mathrm{m}^{-1} \cdot \mathrm{K}^{-1}$ for example), see Fig. 7(b).

Fig. 8 shows the relative deviations between the values of the volumetric heat capacity estimated from the $15 \mathrm{~mm}$ measurement and the nominal values as a function of the Stark number. It shows that for the estimation error to be less than $2 \%$ it is necessary that $N>0.5$ in the case of an absorbing and scattering material, see Fig. 8(a). In the case of a purely scattering material, the Stark number must be greater than 10 for the accuracy to be better than $5 \%$.

It should also be noted that the estimation time interval decreases when the extinction coefficient decreases and this is all the more so as the share of scattering is large. On the one hand the time required for radiation to be well taken into account by a conductive model with effective thermal conductivity increases, and on the other hand, the increase of the effective thermal conductivity when the extinction coefficient $\beta$ decreases makes the assumption of the semi-infinite medium less valid. As an example, the estimation interval for the high density thermal insulating material varies from $0 \mathrm{~s}$ to $1000 \mathrm{~s}$ for $\beta=10000$ at $T=1000^{\circ} \mathrm{C}$ to $80 \mathrm{~s}$ to $250 \mathrm{~s}$ for $\beta=500$ at $T=1500^{\circ} \mathrm{C}$.

\section{Results and discussion}

\subsection{Distance calibration}

The main source of uncertainty in the estimation of the volumetric heat capacity is the uncertainty in the distance $d$ between the heating 


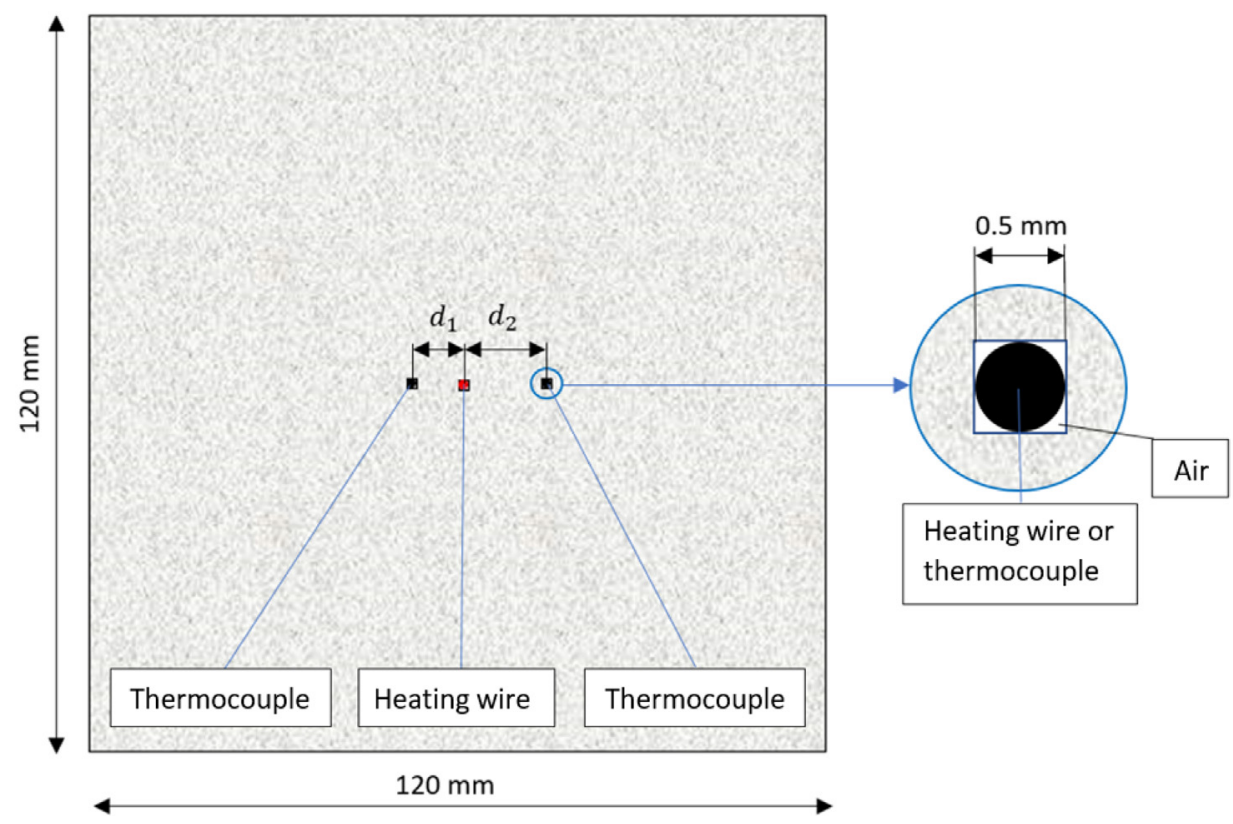

Fig. 6. Diagram of the modeled system with $d_{1}=10 \mathrm{~mm}$ and $d_{2}=15 \mathrm{~mm}$.

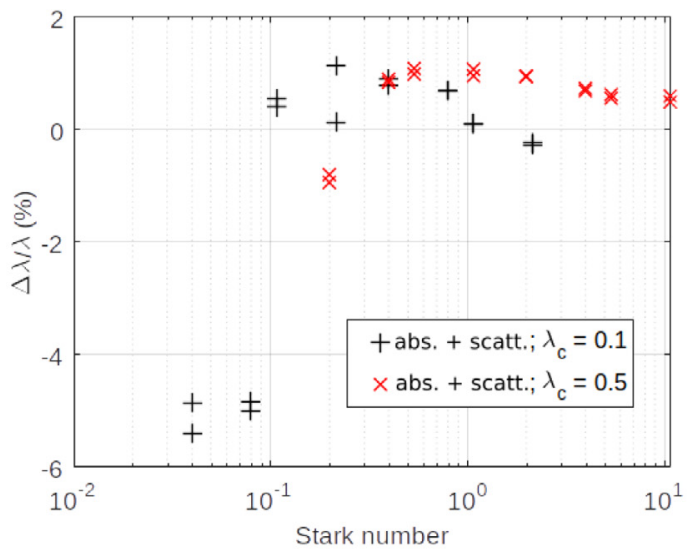

(a)

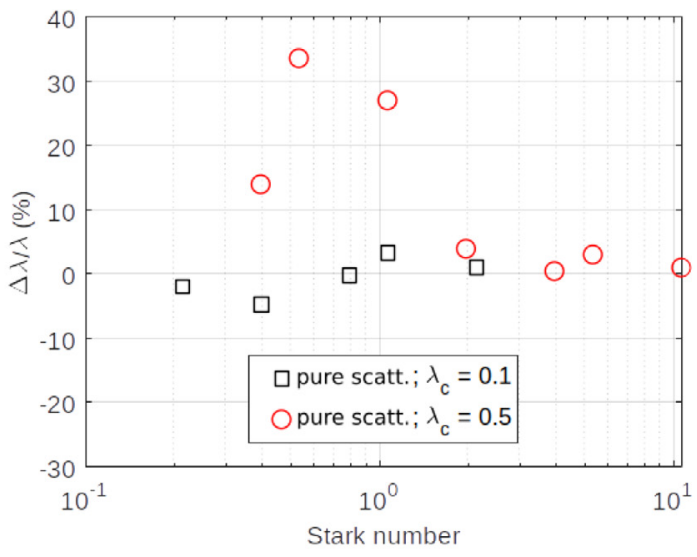

(b)

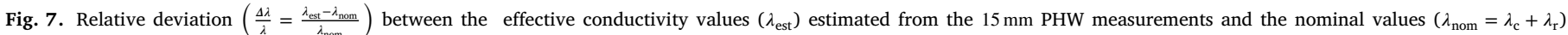
as a function of the Stark number in (a) the absorption-diffusion case and (b) the pure scattering case.

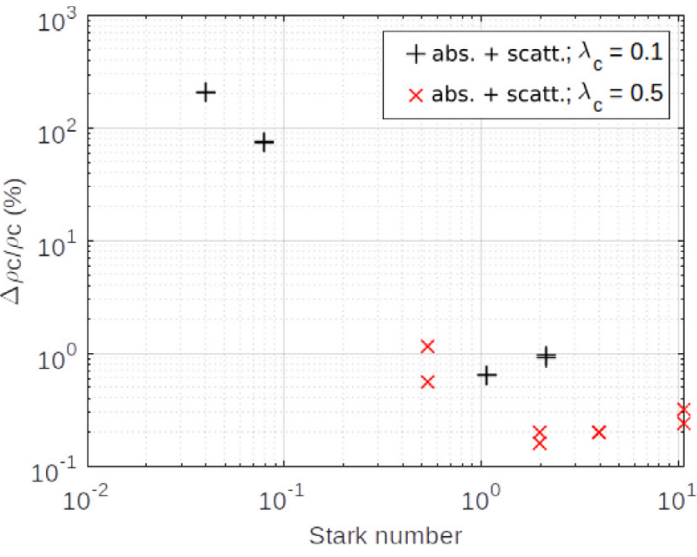

(a)

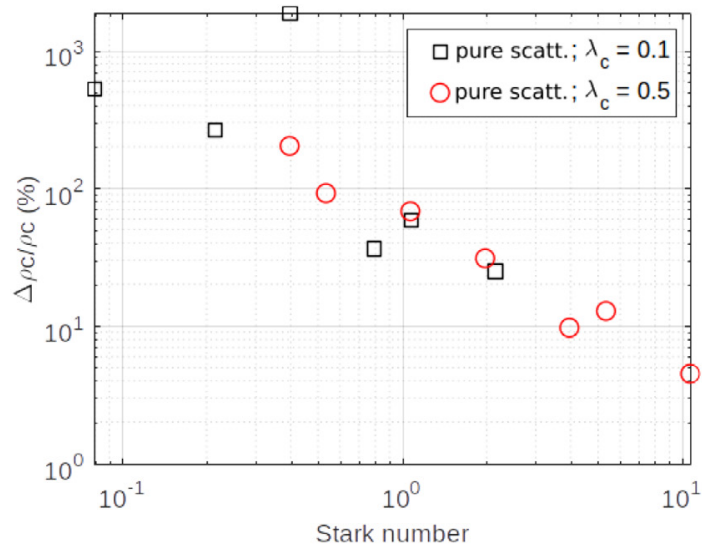

(b)

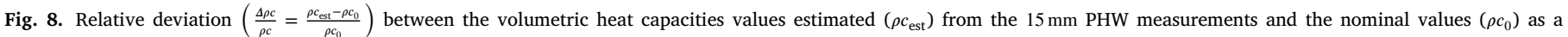
function of the Stark number in (a) the absorption-diffusion case and (b) the pure scattering case. 


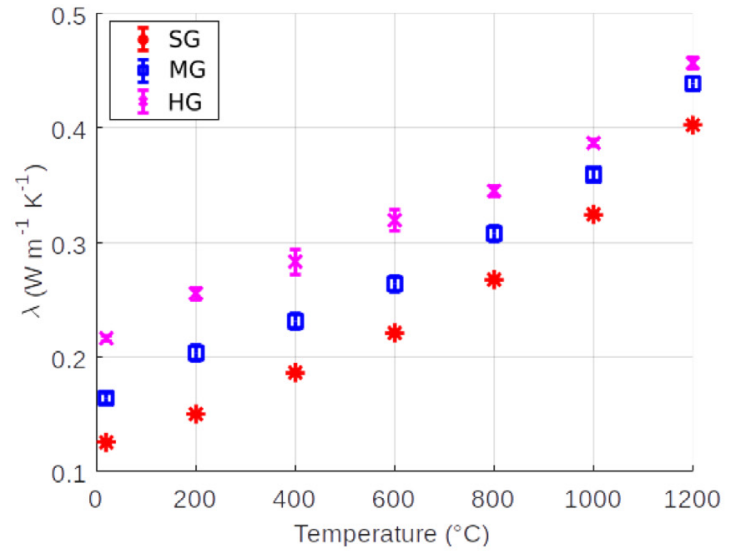

(a)

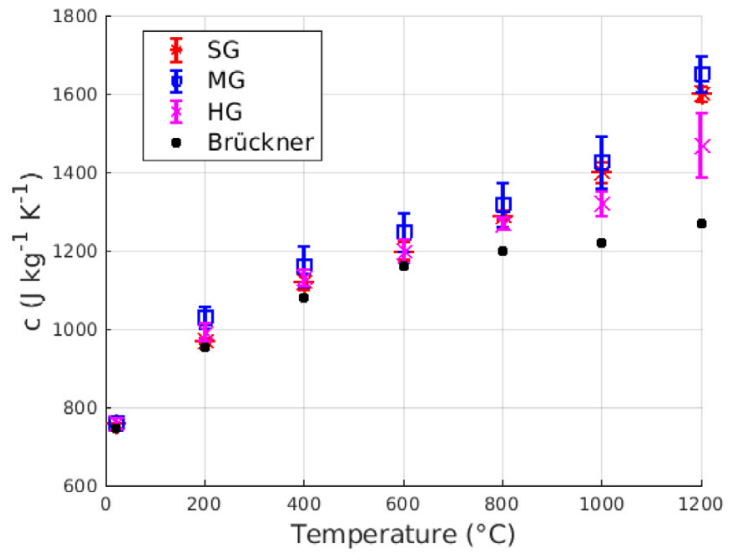

(b)

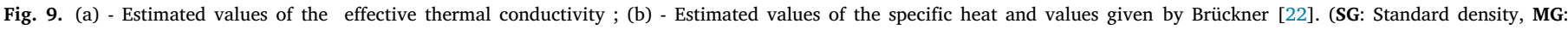
Medium density, HG: High density).

wire and the thermocouple. The uncertainty on this distance is of the order of $0.5 \mathrm{~mm}$ given the brittleness of the materials in which the grooves are made. For a distance $d=10 \mathrm{~mm}$, an uncertainty of $0.5 \mathrm{~mm}$ leads to uncertainties of respectively $5 \%$ on the distance $d$ and $10 \%$ on $\rho c$ [3]. To reduce this uncertainty, we performed three parallel hot-wire experiments at $20^{\circ} \mathrm{C}$ for each of the 3 SilPower ${ }^{\circledR}$ samples which specific heat at $20^{\circ} \mathrm{C}$ was measured by differential scanning calorimetry using the Setaram $\mu \mathrm{dSc}_{3}$ apparatus and we obtained: $c=760 \mathrm{~J}^{-1} \mathrm{~kg}^{-1} \mathrm{~K}^{-1}$. The accuracy of the measurement is estimated at $2 \%$. These experimental curves were used to estimate the unknown parameters $\lambda, d$ and $R$ considering the known value of $\rho c$.

The results are presented in Table 2 . A very good reproducibility of the measurements is observed. It is also observed that the deviation between the estimated values and the nominal values $\left(d_{\text {nom, } 1}=10 \mathrm{~mm}\right.$ and $d_{\text {nom, } 2}=15 \mathrm{~mm}$ ) can reach $5 \%$. Since the uncertainty on $\rho c$ is mainly due to the error on $d$, the estimation error on $\rho c$ is reduced to the uncertainty on the reference value used to estimate the distances, i.e. about $2 \%$. The accuracy of the estimate is thus significantly improved.

\subsection{Measurements on silpower ${ }^{\circledR}$}

The estimated values of $d_{1}$ and $d_{2}$ were then used to estimate $\lambda$, $\rho c$ and $R$ for the experiments performed between $200^{\circ} \mathrm{C}$ to $1200^{\circ} \mathrm{C}$. Consistent with the results of the theoretical study, the values of $\lambda$ and $\rho c$ estimated with the measured temperatures at distances $d_{1}$ and $d_{2}$ from the heating wire were almost identical. The average deviation observed for $\lambda$ is $1.6 \%$ and that for $\rho c$ is $2.1 \%$. The results obtained for $\lambda$ and $\rho c$ are shown in Figs. 9(a) and 9(b) respectively. The points correspond to the average of the 6 measurements performed ( 3 for each distance) and the bars represent the standard deviations of these groups of 6 points. The 21 standard deviations on $\rho c$ calculated each on 6 measurements were all less than $5 \%$ with an average of $2.6 \%$ which demonstrates the effectiveness of the distance calibration procedure.

The values of thermal conductivity increase as expected with temperature and with density. The values of the specific heat $c$ are close for the three materials, which is logical given that they have the same composition. These values are also very close to those measured by Brückner for silica up to $600{ }^{\circ} \mathrm{C}$ [22] also shown in Fig. 9. For temperatures above $800^{\circ} \mathrm{C}$, the difference between our values and those of Brückner increases with temperature, these differences will be explained later after the extinction coefficients of the different grades have been estimated.

Fig. 10 shows the experimental and model curves from the estimated values as well as the estimation residuals $\times 10$ for the SilPower ${ }^{\circledR} \mathrm{SG}$ at $20^{\circ} \mathrm{C}$ and at $1200^{\circ} \mathrm{C}$ for the measurement at $d=10 \mathrm{~mm}$. It can be
Table 2

Estimated values of SilPower ${ }^{\circledR}$ thermal conductivity and heating wire-thermocouple distances at $20^{\circ} \mathrm{C}$.

\begin{tabular}{|c|c|c|c|c|c|c|c|}
\hline & $\mathrm{N}^{\circ} \exp$ & $\lambda$ & $\mathrm{d}_{1}$ & $\frac{d_{1}-d_{\mathrm{nom}, 1}}{d_{\mathrm{nom}, 1}}$ & $\lambda$ & $\mathrm{d}_{2}$ & $\frac{d_{2}-d_{\text {nom }, 2}}{d_{\text {nom }, 2}}$ \\
\hline & & $\mathrm{W} \mathrm{m^{-1 }} \mathrm{K}^{-1}$ & $\mathrm{~mm}$ & $\%$ & $\mathrm{~W} \mathrm{m^{-1 }} \mathrm{K}^{-1}$ & $\mathrm{~mm}$ & $\%$ \\
\hline \multirow{4}{*}{$\rho=581 \mathrm{~kg} \mathrm{~m}^{-3}$} & 1 & 0.123 & 9.99 & & 0.121 & 14.65 & \\
\hline & 2 & 0.124 & 10.00 & & 0.124 & 14.67 & \\
\hline & 3 & 0.125 & 10.01 & & 0.125 & 14.67 & \\
\hline & Mean & 0.124 & 10.00 & 0.0 & 0.123 & 14.66 & 2.27 \\
\hline \multirow{4}{*}{$\rho=741 \mathrm{~kg} \mathrm{~m}^{-3}$} & 1 & 0.169 & 9.72 & & 0.160 & 14.17 & \\
\hline & 2 & 0.169 & 9.72 & & 0.160 & 14.18 & \\
\hline & 3 & 0.168 & 9.71 & & 0.158 & 14.78 & \\
\hline & Mean & 0.169 & 9.72 & 2.80 & 0.159 & 14.17 & 5.53 \\
\hline \multirow{4}{*}{$\rho=910 \mathrm{~kg} \mathrm{~m}^{-3}$} & 1 & 0.213 & 9.38 & & 0.211 & 14.78 & \\
\hline & 2 & 0.217 & 9.43 & & 0.217 & 14.91 & \\
\hline & 3 & 0.214 & 9.46 & & 0.214 & 14.88 & \\
\hline & Mean & 0.215 & 9.42 & 5.80 & 0.214 & 14.85 & 0.96 \\
\hline
\end{tabular}

seen that the residuals are perfectly flat and centered on the estimation interval $[100 \mathrm{~s} ; 800 \mathrm{~s}]$ which validates the model of an effective thermal conductivity on this time domain. The estimation at $20^{\circ} \mathrm{C}$ leads to perfectly flat residuals as early as $40 \mathrm{~s}$ while at $1200^{\circ} \mathrm{C}$ we observe a divergence of the residuals up to $100 \mathrm{~s}$. The estimated values of the temperature shift are $T_{1}=-0.039^{\circ} \mathrm{C}$ at $20^{\circ} \mathrm{C}$ and $T_{1}=1.02^{\circ} \mathrm{C}$ at $1200^{\circ} \mathrm{C}$. The negative value at $20^{\circ} \mathrm{C}$ is due to the contact resistance in the absence of significant radiation at this temperature while the positive value at $1200^{\circ} \mathrm{C}$ is mainly due to the fact that the Rosseland model underestimates the radiation at short times and that the radiation, negligible at $20^{\circ} \mathrm{C}$, becomes significant at $1200^{\circ} \mathrm{C}$.

\subsection{Estimation of the extinction coefficient}

The following assumptions are made:

- The thermal conductivities of the solid and air in the porous medium evolve linearly with temperature.

- The radiation is represented by the Rosseland approximation with a constant extinction coefficient $\beta$.

The effective thermal conductivity can therefore be written:

$\lambda=a+b T+\frac{16 n^{2} \sigma T^{3}}{3 \beta}$ 


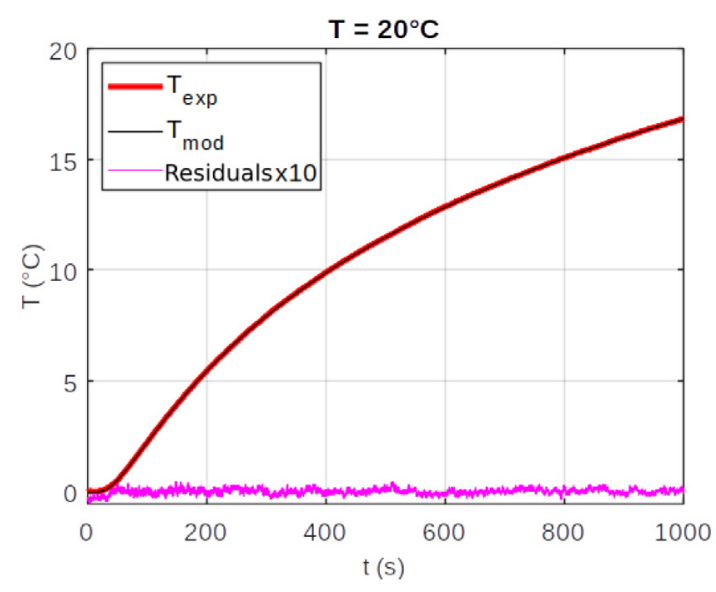

(a)

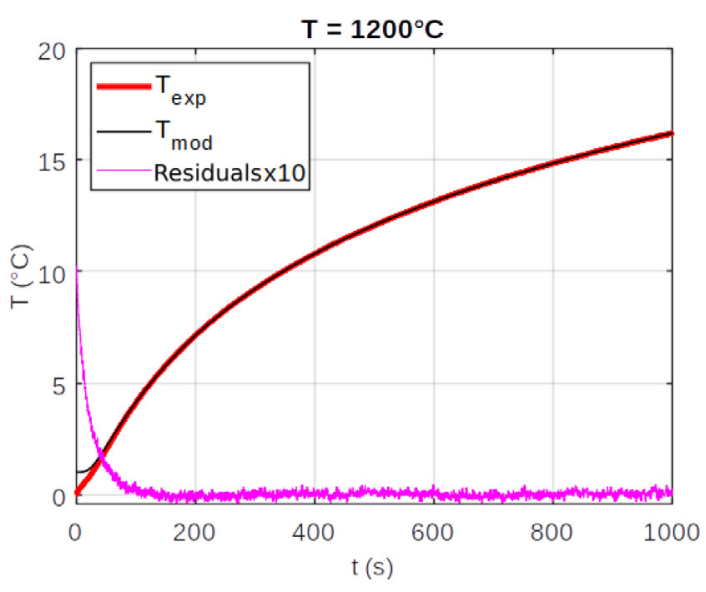

(b)

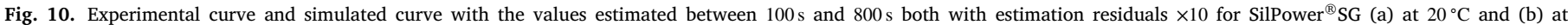
$1200^{\circ} \mathrm{C}$ for $d=10 \mathrm{~mm}$.

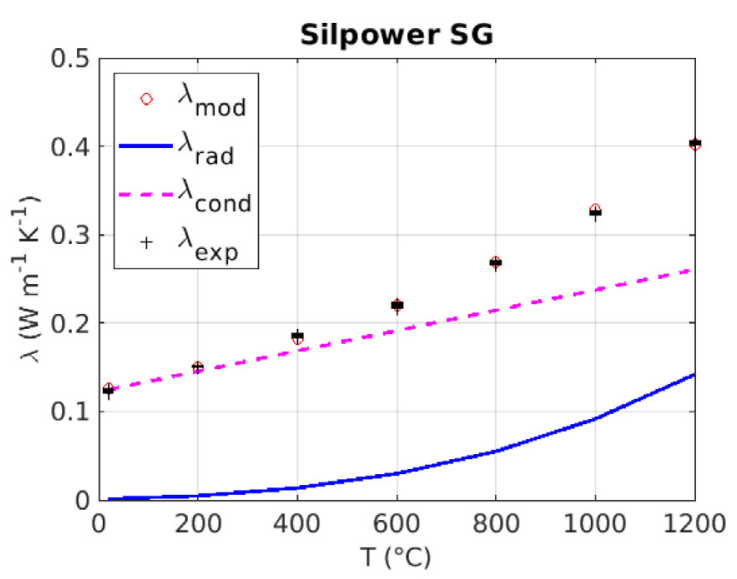

(a) SilPower ${ }^{\circledR}$ SG

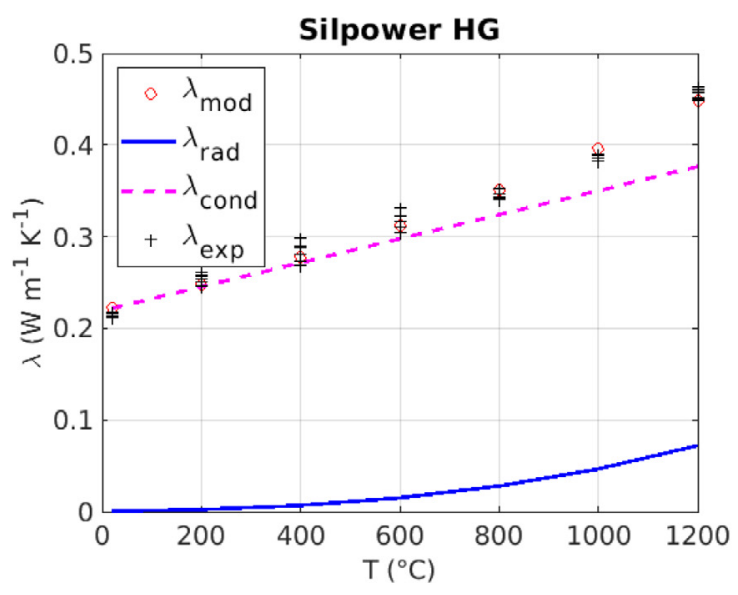

(b) SilPower ${ }^{\circledR}$ HG

Fig. 11. Estimated values of the various conductivities for SilPower ${ }^{\circledR}$ SG (Standard density) and SilPower ${ }^{\circledR}$ HG (High density)

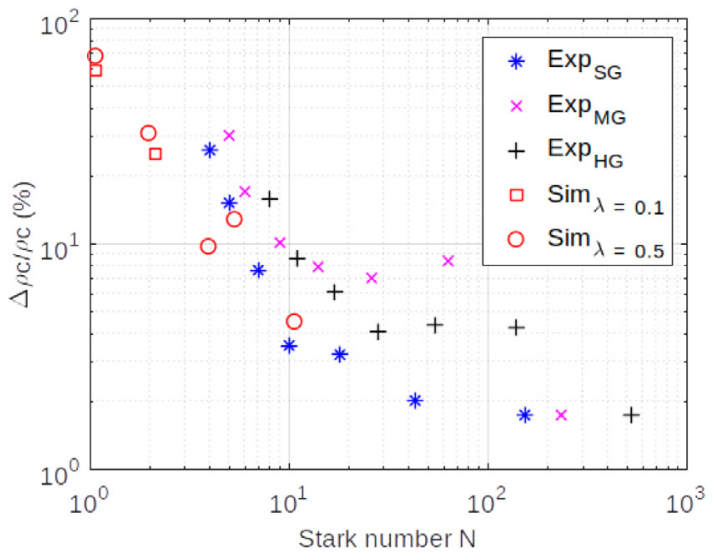

Fig. 12. Relative deviation between the values of the specific heat estimated from experiments or simulations and those given by Brückner [22].

For each material, we can then estimate the parameters $a, b$ and $\beta$ by minimizing the sum:

$S=\sum_{j=1}^{J} \sum_{k=1}^{K}\left[\lambda_{\exp , k}\left(T_{j}\right)-\lambda_{\text {mod }}\left(T_{j}\right)\right]^{2}$
Where $\lambda_{\text {mod }}$ corresponds to Eq. (17), $J$ is the number of temperatures for which the measurement of $\lambda$ was performed and $K$, the number of thermal conductivity estimations carried out at each temperature $T_{j}$. Six estimations were performed at each temperature ( $K=6$ ), respectively three at a distance $d_{1}=10 \mathrm{~mm}$ and three at a distance $d_{2}=15 \mathrm{~mm}$ (see Table 2).

The standard deviation of each parameter $a, b$ and $\beta$ is then deduced during the standard procedure of fitting and given by the covariance matrix. The results are presented in Table 3 and the values of the different estimated conductivities are presented in Fig. 11 for SilPower ${ }^{\circledR}$ SG and SilPower ${ }^{\circledR}$ HG. The following comments can be made:

- The extinction coefficient decreases very logically when the density decreases.

- The values of the effective conductivity calculated with the estimated values of the parameters $a, b$ and $\beta$ coincide perfectly with the experimental values.

- It is often assumed that the thermal conductivity of the porous solid matrix is proportional to that of the solid [23]. Despite the high uncertainties, the ratios of the thermal conductivity of SilPower ${ }^{\circledR}$ at $1200^{\circ} \mathrm{C}$ to its value at $20^{\circ} \mathrm{C}$ (obtained for all three densities) are found to be very close (maximum deviation of $20 \%$ ) to that obtained for silica, of which the backbone of SilPower ${ }^{\circledR}$ is more than $99 \%$ (see Table 3). 
Table 3

Estimated values of parameters $a, b$ and $\beta$ and their uncertainties.

\begin{tabular}{|c|c|c|c|c|c|c|c|c|c|c|}
\hline & $\rho$ & $\beta$ & $\sigma_{\beta}$ & $a$ & $\sigma_{a}$ & $b$ & $\sigma_{b}$ & $\frac{\lambda_{c} 1200^{\circ} \mathrm{C}}{\lambda_{c 2} 20^{\circ} \mathrm{C}}$ & & \\
\hline & $\mathrm{kg} \mathrm{m}^{-3}$ & $\mathrm{~m}^{-1}$ & $\%$ & $\mathrm{~W} \mathrm{m^{-1 }} \mathrm{K}^{-1}$ & $\%$ & $\mathrm{~W} \mathrm{m^{-1 }} \mathrm{K}^{-1}$ & $\%$ & Thermal & $\mathrm{SiO}_{2}{ }^{\mathrm{a}}$ & Air \\
\hline SG & $581 \mathrm{~kg} \mathrm{~m}^{-3}$ & $6.83 \times 10^{3}$ & 3.0 & $9.09 \times 10^{-2}$ & 2.1 & $1.15 \times 10^{-4}$ & 3.2 & 2.4 & & \\
\hline MG & $741 \mathrm{~kg} \mathrm{~m}^{-3}$ & $7.86 \times 10^{3}$ & 8.6 & $1.34 \times 10^{-1}$ & 3.7 & $1.20 \times 10^{-4}$ & 7.6 & 2.0 & 2.0 & 3.5 \\
\hline HG & $910 \mathrm{~kg} \mathrm{~m}^{-3}$ & $1.35 \times 10^{4}$ & 19.4 & $1.83 \times 10^{-1}$ & 3.5 & $1.31 \times 10^{-4}$ & 9.2 & 1.8 & & \\
\hline
\end{tabular}

${ }^{\text {a }}$ From Combis et al. [24].

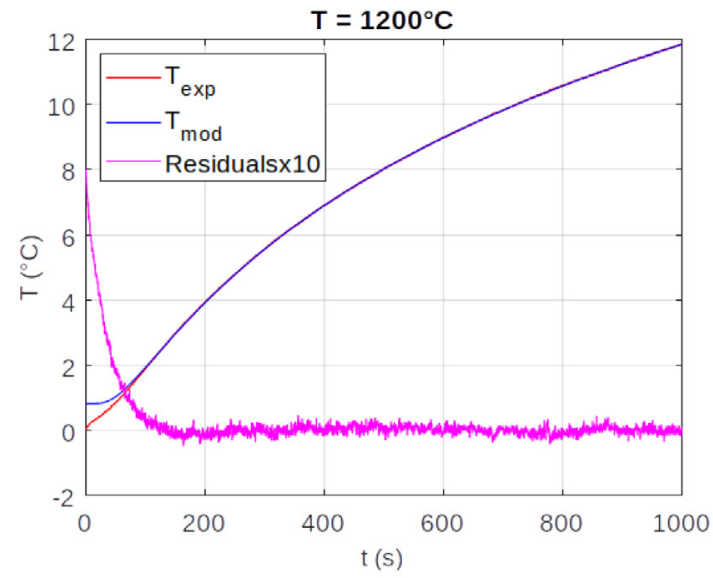

(a)

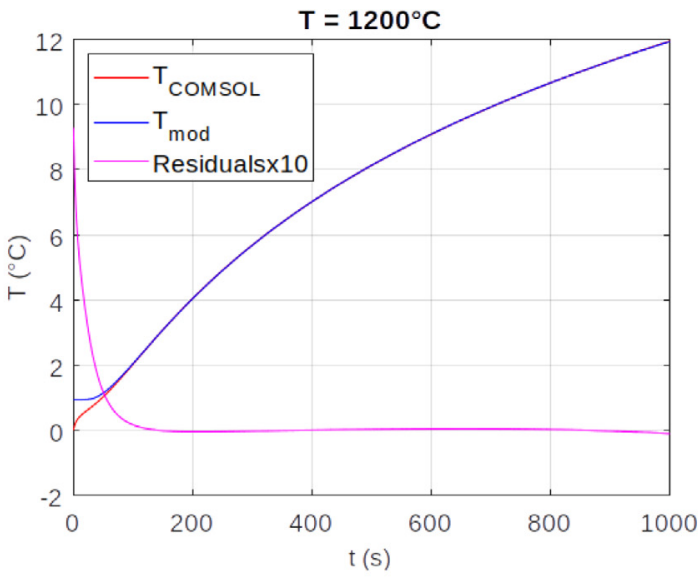

(b)

Fig. 13. (a) - Experimental curve and model curve ; (b) - Simulated curve with COMSOL Multiphysics ${ }^{\circledR}$ and model curve.

- The accuracy of the $\beta$ estimate decreases when the value of $\beta$ increases: this is explained by the fact that when $\beta$ increases, the share of the radiative conductivity $\lambda_{\mathrm{r}}$ in the effective conductivity $\lambda$ decreases so the sensitivity of $\lambda$ to $\beta$ decreases.

Estimates of the extinction coefficients $\beta$ and thermal conductivities $\lambda_{\mathrm{c}}$ of the solid allow the calculation of the Stark number $N$ for each grade and for each temperature. This then allows us to plot the relative differences between the estimated values $\rho c$ and those calculated from the Brückner values as a function of the Stark number $N$ (see Fig. 12). We plotted on the same graph the deviations between the values estimated from COMSOL Multiphysics ${ }^{\circledR}$ simulations for a scattering material $\left(k_{\mathrm{s}}=0.9999 \beta\right)$ and those from Brückner [22]. The deviations calculated from experiments and estimates follow the same trend and are close. SilPower ${ }^{\circledR}$ thus behaves as a highly scattering material towards radiation which is consistent with its properties: it is a highly reflective material (reflectivity greater than $95 \%$ in the infrared [25]) and it contains a volume fraction of $60 \%$ to $70 \%$ of air (transparent) depending on the density.

Finally, as an example, a COMSOL Multiphysics ${ }^{\circledR}$ simulation was performed with the estimated parameters for SilPower ${ }^{\circledR} \mathrm{SG}$ at $1200^{\circ} \mathrm{C}$ i.e. $\beta=6920 \mathrm{~m}^{-1} ; \lambda_{\mathrm{r}}=0.140 \mathrm{~W} \mathrm{~m}^{-1} \mathrm{~K}^{-1} ; \lambda_{\mathrm{c}}=\lambda-\lambda_{\mathrm{r}}=$ $0.263 \mathrm{~W} \mathrm{~m}^{-1} \mathrm{~K}^{-1}$ and considering a highly scattering material $\left(k_{\mathrm{s}}=\right.$ $0.9999 \beta)$. This simulation was then treated as an experimental curve to estimate $\lambda$ and $\rho c$. The value of $\rho c$ was adjusted to $\rho c=7.00 \times$ $10^{5} \mathrm{~J} \mathrm{~m}^{-3} \mathrm{~K}^{-1}$ so that the estimations from the experimental and the simulated curves lead to the same value, $\rho c=8.30 \times 10^{5} \mathrm{~J} \mathrm{~m}^{-3} \mathrm{~K}^{-1}$.

Fig. 13(a) shows one experimental curve obtained at $1200^{\circ} \mathrm{Celsius,}$ the model curve with the estimated parameters and the estimation residuals. Fig. 13(b) shows the curve simulated with COMSOL Multiphysics ${ }^{\circledR}$, the model curve plotted with the estimated parameters and the estimation residuals.

We can see that the residuals have globally the same shape and an amplitude of the same order of magnitude as those represented on Fig. 9(b), which confirms the very scattering character of SilPower ${ }^{\circledR}$. Moreover, the deviation between the nominal and estimated values of $\rho c$ which is $\frac{\Delta \rho c}{\rho c}=\frac{8.3-7.0}{8.3}=15.6 \%$ is of the same order of magnitude as the one observed with the experiments.

Nevertheless, the value of the absorption coefficient used to match the COMSOL Multiphysics ${ }^{\circledR}$ simulation and the experiment is not realistic $\left(k_{\mathrm{a}}=6.9 \mathrm{~m}^{-1}\right)$. This may be due to the approximation of the P1 model or to the hypothesis of a gray medium. It will therefore be necessary to develop a more accurate model if we want to represent the whole experimental curve in order to estimate for example the extinction or diffusion coefficient of the medium.

\section{Conclusion}

The influence of the radiation heat transfer on the estimation of the thermal conductivity of a semi-transparent material by the parallel hot-wire method has been first investigated with COMSOL Multiphysics ${ }^{\circledR}$ using the coupled conduction-radiation model based on the P1 approximation for the radiation calculation. It was shown that the thermal conductivity may be estimated with an accuracy better than $2 \%$ if the Stark number $N$ (representing the ratio conduction/radiation) is:

- Greater than 0.1 for a purely absorbing or absorbing and scattering medium.

- Greater than 2 for a purely scattering medium.

It has also been shown that the classical estimation method based on the estimation of the three parameters $(\lambda, \rho c)$ leads to negative values of the thermal contact resistance $R$ ( which makes no physical sense) when radiation increases and creates an estimation bias. An new estimation method has been proposed to avoid this estimation bias.

A calibration process of the distance between the hot wire and the thermocouple has also been proposed and validated, enabling a more accurate estimation of the volume heat capacity. An experimental study carried out on three insulating materials with densities ranging from $581 \mathrm{~kg} \mathrm{~m}^{-3}$ to $910 \mathrm{~kg} \mathrm{~m}^{-3}$ and at temperatures ranging from $20^{\circ} \mathrm{C}$ to $1200^{\circ} \mathrm{C}$ confirms the results of the theoretical study. A method 
enabling the estimation of the extinction coefficient from thermal conductivity measurements at various temperatures has been presented and successfully applied to the three tested materials.

\section{Declaration of competing interest}

The authors declare that they have no known competing financial interests or personal relationships that could have appeared to influence the work reported in this paper.

\section{Data availability}

Data will be made available on request.

\section{References}

[1] Y. Jannot, A. Degiovanni, An improved model for the parallel hot wire: Application to thermal conductivity measurement of low density insulating materials at high temperature, Int. J. Therm. Sci. 142 (2019) 379-391.

[2] Y. Jannot, A. Degiovanni, V. Schick, J. Meulemans, Apparent thermal conductivity measurement of anisotropic insulating materials at high temperature by the parallel hot-wire method, Int. J. Therm. Sci. 160 (2021) 106672.

[3] ISO 18894-2:2007, Refractory Materials- Determination of Thermal ConductivityPart 2: Hot-Wire Method (Parallel), Standard, International Organization for Standardization, Geneva, CH, 2007.

[4] Y.Z. Zhang, S.X. Cheng, J.A. Lee, X.S. Ge, Simultaneous measurement of thermal conductivity and thermal diffusivity of solids by the parallel-wire method, Int. J. Thermophys. 12 (3) (1991) 577-584.

[5] G. Grazzini, C. Balocco, U. Lucia, Measuring thermal properties with the parallel wire method: A comparison of mathematical models, Int. J. Heat Mass Transfer 39 (10) (1996) 2009-2013.

[6] W.N. dos Santos, R. Gregório, Numerical and experimental determination of minimum and maximum measuring times for the hot wire parallel technique, Cerâmica 49 (2003) http://dx.doi.org/10.1590/S0366-69132003000100007.

[7] H.-P. Ebert, J. Fricke, Influence of radiative transport on hot-wire thermal conductivity measurements, High Tempe. High Press. (Print) 30 (6) (1998) 655-669.

[8] U. Gross, L.-T.-S. Tran, Radiation effects on transient hot-wire measurements in absorbing and emitting porous media, Int. J. Heat Mass Transfer 47 (14-16) (2004) 3279-3290.

[9] R. Coquard, D. Baillis, D. Quenard, Experimental and theoretical study of the hot-wire method applied to low-density thermal insulators, Int. J. Heat Mass Transfer 49 (23-24) (2006) 4511-4524.

[10] N. Daouas, A. Fguiri, M.-S. Radhouani, Solution of a coupled inverse heat conduction-radiation problem for the study of radiation effects on the transient hot wire measurements, Exp. Therm Fluid Sci. 32 (8) (2008) 1766-1778.
[11] H. Zhang, Y.X. Ma, X. Wang, G.H. Tang, Numerical study of the influence of thermal radiation on measuring semi-transparent thermal insulation material with hot wire method, Int. Commun. Heat Mass Transfer (ISSN: 0735-1933) 121 (2021) 105120, http://dx.doi.org/10.1016/j. icheatmasstransfer.2021.105120, URL https://www.sciencedirect.com/science/ article/pii/S0735193321000142.

[12] F.R. De Hoog, J.H. Knight, A.N. Stokes, An improved method for numerical inversion of Laplace transforms, SIAM J. Sci. Stat. Comput. 3 (3) (1982) 357-366.

[13] Michael F. Modest, Chapter 16 - the method of spherical harmonics (PNapproximation), in: Michael F. Modest (Ed.), Radiative Heat Transfer (Third Edition), third ed., Academic Press, Boston, ISBN: 978-0-12-386944-9, 2013, pp. 495-540, http://dx.doi.org/10.1016/B978-0-12-386944-9.50016-9, URL https: //www.sciencedirect.com/science/article/pii/B9780123869449500169.

[14] J.R. Howell, M.P. Menguc, R. Siegel, Thermal Radiation Heat Transfer, fifth ed., CRC Press, ISBN: 9781439894552, 2010, URL https://books.google.fr/books?id= FBjSBQAAQBAJ.

[15] R.E. Marshak, Note on the spherical harmonic method as applied to the milne problem for a sphere, Phys. Rev. 71 (1947) 443-446, http://dx.doi.org/10.1103/ PhysRev.71.443, URL https://link.aps.org/doi/10.1103/PhysRev.71.443.

[16] Y. Jannot, A. Degiovanni, Thermal Properties Measurement of Materials, John Wiley \& Sons, 2018.

[17] D.W. Marquardt, An algorithm for least-squares estimation of nonlinear parameters, J. Soc. Ind. Appl. Math. 11 (2) (1963) 431-441.

[18] Heat Transfer Module User's Guide, 2020, URL https://doc.comsol.com/5.6 doc/com.comsol.help.heat/HeatTransferModuleUsersGuide.pdf, "Theory for Radiation in Participating Media, P1 Approximation Theory", 268-271, COMSOL Multiphysics ${ }^{\text {v }}$ v. 5.6.

[19] Y. Jannot, A. Degiovanni, V. Schick, J. Meulemans, Thermal diffusivity measurement of insulating materials at high temperature with a four-layer (4L) method, Int. J. Therm. Sci. 150 (2020) 106230.

[20] R. Viskanta, Heat transfer by conduction and radiation in absorbing and scattering materials, J. Heat Transfer 87 (1) (1965) 143-150.

[21] C. Cintolesi, H. Nilsson, A. Petronio, V. Armenio, Numerical simulation of conjugate heat transfer and surface radiative heat transfer using the $\mathrm{P} 1$ thermal radiation model: Parametric study in benchmark cases, Int. J. Heat Mass Transf. 107 (2017) 956-971, http://dx.doi.org/10.1016/j.ijheatmasstransfer.2016.11. 006, URL https://hal.archives-ouvertes.fr/hal-02070285.

[22] R. Brueckner, Properties and structure of vitreous silica. I, J. Non-Crystal. Solids 5 (2) (1970) 123-175

[23] M. Rottmann, T. Beikircher, H.-P. Ebert, Thermal conductivity of evacuated expanded perlite measured with guarded-hot-plate and transient-hot-wire method at temperatures between $295 \mathrm{~K}$ and $1073 \mathrm{~K}$, Int. J. Therm. Sci. 152 (2020) 106338

[24] P. Combis, P. Cormont, L. Gallais, D. Hebert, L. Robin, J.-L. Rullier, Evaluation of the fused silica thermal conductivity by comparing infrared thermometry measurements with two-dimensional simulations, Appl. Phys. Lett. 101 (21) (2012) 211908.

[25] SilPower ${ }^{\circledR}$ standard and high density, 2021, https://www.quartz.saint-gobain. com/sites/imdf.quartz.com/files/silpower_leaflet_76014.pdf. (Accessed 14 December 2021) 